\title{
Research Progress of Artificial Psychology and Artificial Intelligence in India
}

\author{
Dr. Janhvi Srivastava *, Priyanka Srivastava ${ }^{* *}$ \\ * Assistant professor, Veer Bahadur Singh Purvanchal University,Jaunpur \\ ${ }^{* *}$ Department Of Computer Science, B.H.U., Varanasi \\ DOI: $10.29322 /$ IJSRP.10.02.2020.p9803 \\ http://dx.doi.org/10.29322/IJSRP.10.02.2020.p9803
}

\begin{abstract}
Artificial Psychology is a discipline that uses information science to achieve a more comprehensive coverage of human psychological activity with artificial machine like: computer and algorithmic models. In the field of information science, researchers have regarded the imitation of human brain, human intelligence, and human behavior as important research directions and contents. A review of automation science and technology shows that more people use brain science, psychology, neuroscience or cybernetics and theory of controlled source in studying algorithms

Artificial Intelligence refers to the ability of machines to perform cognitive tasks like thinking, perceiving, learning, problem solving and decision making. Initially conceived as a technology that could mimic human intelligence, AI has evolved in ways that far exceed its original conception.
\end{abstract}

Index Terms- Artificial Intelligence, Artificial Psychology, Deep Learning, Machine Learning.

\section{INTRODUCTION}

A fter decades of research, today AI has topped the heights of achievements in technology.

With incredible advances made in data collection, processing and computation power, intelligent systems can now be deployed to take over a variety of tasks, enable connectivity and enhance productivity. As AI's capabilities have dramatically expanded, so have its utility in a growing number of fields.

The truly transformative nature of the technology, yet the nascent stage of its adoption worldwide, provides India with an opportunity to define its own brand of AI leadership. \#AI for All the brand proposed for India implies inclusive technology leadership, where the full potential of AI is realized in pursuance of the country's unique needs and aspirations. The strategy should strive to leverage AI for economic growth, social development and inclusive growth, and finally as a Garage for emerging and developing economies.

Adoption of AI across the value chain viz. startups, private sector, PSUs and government entities, will truly unlock the potential by creating a virtuous cycle of supply and demand. The barriers to AI development and deployment can effectively be addressed by adopting the marketplace model - one that enables market discovery of not only the price but also of different approaches that are best suited to achieve the desired results. A three-pronged, formal marketplace could be created focusing on data collection and aggregation, data annotation and deployable models.

The content of artificial intelligence research focuses on how to express, acquire, and use knowledge. Artificial intelligence, however, is only the preliminary stage in the field of personification research because human psychological activities include feeling, perception, memory, thinking, emotion, will, character, and creation. Among these activities, only feeling, perception, memory, and thinking are encompassed by artificial intelligence; thus, its scope is limited. Therefore, to study artificial psychology and artificial emotion and develop cutting-edge technology and industrial applications in these fields, artificial intelligence research (research results and research methods) must be used as theoretical basis; new theories and methods in psychology, brain science, neuroscience, information science, computer science, and automation science should be integrated; and psychological activities, particularly emotion, will, character, and creation, should be simulated .

Two CLOSELY RELATED ASPECTS of Artificial Intelligence that have received comparatively little attention in the recent literature are research methodology and the analysis of computational techniques that span multiple application areas. We believe both issues to be increasingly significant, as Artificial Intelligence matures into a science and spins off major application efforts. It is imperative to analyze the repertoire of AI methods with respect to past experience, utility in new domains, extensibility, and functional equivalence with other techniques, if $\mathrm{N}$ is to become more effective in building upon prior results rather than continually reinventing the proverbial wheel. Similarly, awareness of research methodology issues can help plan future research by learning from past successes and failures. We view the study of research methodology to be similar to the analysis of operational AI techniques, but at a meta-level; that is, research methodology analyzes the techniques and methods used by the researchers themselves, rather than their programs, to resolve issues of selecting interesting and tractable problems to investigate, and of deciding how to proceed with their investigations. A public articulation of methodological issues that typically remain implicit in the literature may provide some helpful orientation for new researchers and broaden the perspective of many AI practitioners.

AI assistants are set to transform the way humans and machines interact:

Convenient and time saving 
Reduction in mundane task

Smarter customer service

Better personalization in interaction

A majority of the participants (60\%) are of the opinion that AI would help furthering social causes and enable human beings to live more fulfilling lives. These include stimulating economic growth, enhancing global health and well-being, improving cyber security and improving efficiencies in imparting education.

- People expect their interactions with digital assistants to expand from being convenience-driven to the point of handling major responsibilities such as that of a teacher or advisor.

- Business decision makers deem AI powered solutions such as machine learning, virtual private assistants, decision support systems, automated data analysts and others to be have a high impact on their businesses in the future.

- The majority (75\%) of business decision makers would prefer either purely AI advisors or a combination of AI and human advisors to make their promotion decisions.

- When it comes to matters of personal health check-ups (77\%) and education $(61 \%)$, the participants are still inclined towards the involvement of human experts. Similar sentiments are echoed regarding the potential loss of human touch associated with AI run customer service.

Artificial Intelligence is intelligence demonstrated by machine or computers in terms of: thinking, reasoning and selfcorrection.

The most prominent types of Artificial Intelligence that have been influencing the rise in technology are Machine Learning (ML) and Deep Learning (DL). There are numerous sectors where Artificial Intelligence is applied.

1. Retail Industry: E-commerce sites are adopting Deep Learning (DL) to help shoppers to easily find what they want to purchase. Online shopping today is a popular and easy mode of shopping for anyone who is able to access to the internet and can operate a smart phone, tablet or a computer. Online shopping websites use Artificial Intelligence (AI) to provide recommendations for the next purchase, depending on previous purchases made by the customer, through algoriothms and search patterns. With the help of AI, online shopping can be made more personal and exclusive. Amazon is planning to use this technology in its new Amazon Go grocery stores.

2. Entertainment Industry: AI technology is applied to optimize video fluency and definition. Online media streaming or video-on-demand services have a built-in AI feature, where recommendations are given to the customer depending on the type of media that has been streamed before or the type of movies watched earlier.

3. Automotive Industry: In recent times, Artificial Intelligence is severely being used for automotive industries across the world. The Automotive industry has developed numerous advanced features through the use of AI. Features such as self-parking, blind spot monitoring and voice recognition are aided by the use of AI. Apps like Uber, Ola and commercial flights that use autopilot are the best example of sectors where AI is being effectively implemented.

4. Healthcare Industry: Artificial Intelligence (AI) helps to analyze data and reports from a patient's file to help select the customized treatment. IBM Watson was developed as a question-answering computer, which has advance AI capabilities such as natural language processing, automated reasoning and others. IBM Watson's first commercial application is its use in utilization management decisions for lung cancer treatment at Memorial Sloan-Kettering Cancer Centre.

5. Manufacturing Industry: Big companies like GE and Siemens are majorly focused on applying Artificial Intelligence to create a comprehensive manufacturing process. The manufacturing industry may be considered as the first use case for the application of AI, using robots for assembly, packing and the likes.

6. Online Customer Service: AI in the customer service space is currently being used to handle some of the monotonous work and is making it easier for human agents to deal with the more difficult issues. These systems are able to handle more requests without human involvement as they have ability to learn by themselves. Several websites today offer the possibility to chat with customer service representatives. Most of the times these are not human, but chat bots that are trained to respond and extract information based on the customer's query.

7. Home Appliances: All smart home appliances which incorporate Internet of Things (IoT) technology make use of AI. The smart home appliances learn the usage pattern of the user over a period of time and then operate themselves through simple commands. One of the examples of AI integrated home appliances is Amazon's Alexa - the virtual assistant developed by Amazon.

8. Smart Phones: Virtual personal assistants such as Siri, Google Now, Google Assistant and Cortana are few examples of AI implementation in smart phones. These virtual personal assistants help you dial a number; locate your favorite restaurant, etc.

9. Banking and Finance: ICICI Bank has been experimenting with AI, through which their email sorts out customer and distributor emails, based on the status of transaction. Robots utilizing AI are quicker at data crunching to provide forecast using market data. Applications would include indicating change in stock trends, managing finances and recommendation of which stocks to buy or sell depending on the customer pattern of purchases or sale.

10. Journalism: News channels and news reporting companies use AI to prepare simple reports on sports and elections which otherwise would be a time consuming exercise. Media houses like the BBC, The New York Times and The Washington Post have been integrating AI into their newsrooms. 
TABLE 1

General research status of Artificial Intelligence in India till 2019:

\begin{tabular}{|l|l|}
\hline DATABASE & ARTIFICIAL INTELLIGENCE \\
\hline ACADEMIC JOURNALS & 12135 \\
\hline DOCTORATE THESIS & \\
& 386 \\
\hline
\end{tabular}

Note: Based on the data sited on Wikipedia.

[5] Z.L. Wang, Artificial Psychology, Mechanical Industry Press, Beijing, 2007.

\section{REFERENCES}

[1] https://www.pwc.in/consulting/technology/data-and-analytics/artificialintelligence-in-india-hype-or-reality.html

[2] https://niti.gov.in/writereaddata/files/document_publication/NationalStrateg y-for-AI-Discussion-Paper.pdf

[3] https://www.aaai.org/ojs/index.php/aimagazine/article/view/371

[4] https://www.weschoolbangalore.in/top-10-applications-of-artificialintelligence/

\section{AUTHORS}

First Author - Dr. Janhvi Srivastava, Assistant Professor, Veer Bahadur Singh Purvanchal University, Jaunpur, drjanhvisrivastava@gmail.com.

Second Author - Priyanka Srivastava, M.SC, Department Of Computer Science, B.H.U, Varanasi, priyankasrivastava658@gmail.com. 